first (i.e. without attempting direct vision internal urethrotomy [DVIU]), and urethroplasty after one, two, or three failed DVIUs. The incremental cost-effectiveness ratio represents the additional cost needed to achieve one successfully voiding patient. Procedure success rates $(95 \%$ for urethroplasty and $50 \%$ for first DVIU) were calculated from published data.

Although research has shown that almost three-quarters of urologists believe that urethroplasty is indicated for short bulbar urethral strictures only after multiple failed DVIUs, this study shows that the most cost-effective strategy is to proceed with urethroplasty if the first DVIU attempt fails. Sensitivity analysis revealed that this strategy is more cost-effective than urethroplasty first, if society is willing to pay $\$ 50,000$ per additional successfully voiding patient and the success rate of first DVIU is $>27.5 \%$. Urethroplasty as a primary treatment is cost-effective only if the expected success rate of a first DVIU is $<35 \%$ (as it is for patients with longer strictures).

The authors highlight the importance of prospective, multicenter studies of the outcomes of urethroplasty and DVIU in the improvement of cost-effectiveness models.

Original article Wright JL et al. (2006) What is the most costeffective treatment for 1 to 2 -cm bulbar urethral strictures: societal approach using decision analysis. Urology 67: 889-893

\section{A comparison of treatment strategies for stage T4 prostate cancer}

Newly diagnosed clinical stage T4 (cT4) prostate cancer is rare, and there is a corresponding lack of data on the efficacy of the various treatment strategies. Johnstone et al. have published an analysis of data from the Surveillance, Epidemiology, and End Results (SEER) database, which compared the survival of patients with cT4 prostate cancer who underwent radical prostatectomy (alone or in combination with another therapy), radiotherapy, hormone therapy, radiotherapy plus hormone therapy, or no treatment at all.

Johnstone et al. determined the 1, 3 and 5-year observed and relative survival rates for over 1,000 patients, who were grouped by treatment type. The relative survival rate was defined as the proportion of patients alive in each time interval, divided by the expected survival rate of a similar group in the population. After controlling for age, ethnicity, tumor grade, SEER registry, clinical tumor extension, year of diagnosis, and regional lymph-node involvement, only three statistically significant differences were found: radical prostatectomy was associated with overall and relative survival rates significantly higher than those for hormone therapy only, radiotherapy only, and no treatment. The benefit of radical prostatectomy, however, seems limited to patients with regional lymph-node extension, and adjuvant therapy does not seem to confer any additional benefit.

The authors suggest that, in terms of survival, radical prostatectomy for selected patients with cT4 prostate cancer is comparable to radiotherapy plus hormone therapy, and is superior to radiotherapy or hormone therapy alone.

Original article Johnstone PAS et al. (2006) Radical prostatectomy for clinical T4 prostate cancer. Cancer 106: 2603-2609

\section{First nomograms proposed for survival of penile squamous cell carcinoma}

The low incidence of squamous cell carcinoma of the penis means that most published studies have included few patients. This limitation has subsequently hampered the identification of prognostic factors, and makes evidence-based treatment problematic. The GUONE (Northeast Uro-oncological Group) database was established in 2004 to collect retrospective clinical and pathologic data on squamous cell carcinoma of the penis from as far back as 1980. Kattan et al. analyzed data from 175 patients included in this database who had undergone partial or total penectomy, in order to construct a nomogram that could predict cancer-specific survival.

Multivariate analyses generated two possible models. The first estimated 5-year cancerspecific survival probabilities on the basis of the pathologic findings of the primary tumor after penectomy and the clinical stage of groin lymph nodes. The authors suggest that this model could be useful for predicting survival after surgical removal of the primary tumor, regardless of the therapy chosen for locoregional lymph nodes.

The second model also estimated survival on the basis of the pathologic findings of the primary tumor after penectomy, but it included 